\title{
Erratum - Alargamiento sobre clavo endomedular en extremidades inferiores - Serie de casos clínicos
}

\section{Erratum - Lower Limb Lengthening Over an Intramedullary Nail - Case Series}

Alejandro Baar Zimend ${ }^{1,2}$ Francisco López Quezada ${ }^{3}$

${ }^{1}$ Jefe Equipo, Ortopedia y Traumatología Infantil, Unidad Ortopedia y

Traumatología, Clínica Las Condes, Santiago, Chile

Address for correspondence Francisco López Quezada, MD, Lo

2 Profesor Asistente, Ortopediia y Traumatología, Universidad de Fontecilla 441, Las Condes, Santiago, Chile, Edificio 4, 4to piso, Depto Chile, Santiago, Chile

${ }^{3}$ Residente, Ortopedia y Traumatología, Universidad de Chile,

Santiago, Chile

Rev Chil Ortop Traumatol 2017;58:76.

\section{ERRATUM}

Rio de Janeiro, 12 de Abril de 2017

Estimados lectores,

En el artículo Alargamiento sobre clavo endomedular en extremidades inferiores, publicado en Rev Chil Ortop Traumatol 2017;58:21-27 (doi: 10.1055/s-0037-1602180), donde se lee: Alejandro Baar Zimend ${ }^{1,2}$ Francisco López Quezada ${ }^{2,3}$

Debería ler:

Alejandro Baar Zimend ${ }^{1,2}$ Francisco López Quezada ${ }^{3}$

Rio de Janeiro, April 12, 2017

Dear readers,

In the article Lower Limb Lengthening Over an Intramedullary Nail, published in Rev Chil Ortop Traumatol 2017;58:21-27 (doi: 10.1055/s-0037-1602180), where it reads:

Alejandro Baar Zimend ${ }^{1,2}$ Francisco López Quezada ${ }^{2,3}$

It should read:

Alejandro Baar Zimend ${ }^{1,2}$ Francisco López Quezada ${ }^{3}$
Copyright ( 2017 by Thieme Revinter Publicações Ltda, Rio de Janeiro, Brazil 